\title{
Mediación y arbitraje en el deporte mexicano
}

\author{
Mediation and arbitration in Mexican sports
}

Daniel Ramiro Coral Muñoz ${ }^{1}$

${ }^{1}$ Facultad de Derecho Universidad Autónoma de Yucatán (UADY)

\author{
Detalles del artículo: \\ Número de palabras: 3695; Tablas: 0; Figuras: 0; Referencias: 20 \\ Recibido: diciembre 2020; Aceptado: enero 2021; Publicado: abril 2021 \\ Conflicto de interés: El autor declara que no existen conflictos de interés. \\ Correspondencia del autor: Daniel Ramiro Coral Muñoz, danielcoralm@ hotmail.com
}

\section{Resumen}

Introducción: Es un hecho innegable que la practica deportiva en México es realizada en abundancia a distintos niveles, desde popular hasta el alto rendimiento o profesional. Y a pesar de ello, pocos son los que realmente conocen a las instancias rectoras de esta actividad, así como a la Comisión de apelación y arbitraje del deporte. Objetivos: El principal objetivo del presente escrito es realizar un análisis de dos medios alternos de solución de controversias, que pueden ser aplicables de manera eficaz en la materia deportiva: la mediación y el arbitraje. Método: Para ello, se realizará un estudio descriptivo de estos conceptos, introduciendo las distintas definiciones y principios fundamentales que rigen dichos procedimientos. De igual manera se realiza un breve análisis de la legislación mexicana en materia deportiva (Ley general de cultura física y deporte) describiendo el régimen jurídico de la mediación y arbitraje dentro de la normativa mexicana. Se describirá la naturaleza de la comisión de apelación y arbitraje del deporte, órgano mexicano de impartición de justicia. Seguidamente se expondrán diversas problemáticas que esta comisión afronta de acuerdo con teorías de diversos juristas especialistas en materia deportiva dentro de México, así como posibles soluciones que se proponen para resolver dichas problemáticas. Resultados: La mediación y el arbitraje aplicados en el deporte son una solución eficaz por excelencia a las problemáticas que surgen en el ámbito deportivo. La comisión de apelación y arbitraje del deporte, principal órgano de impartición de "justicia deportiva" en México, en su normativa reconoce distintos tipos de procedimientos como la mediación o el arbitraje, no obstante, a esta situación, son muy limitados los casos llevados ante ella. Conclusiones: Esta comisión debería contar con mayores facultades de coercitividad para poder funcionar de manera eficaz en México, toda vez que al no carecer de fuerza vinculatoria propia genera una sensación de "simulación" en su proceder.

Palabras clave: Derecho, Mecanismos Alternos de Solución de Controversias, Derecho Deportivo. 


\section{Abstract}

Introduction. It is an undeniable fact that sport practice in Mexico is done in abundance at different levels, from popular to high performance or professional. And in spite of this, few are those who really know the governing bodies of this activity, as well as the Sports Appeal and Arbitration Commission. Objetives. The main objective of this paper is to analyze two alternative means of dispute resolution, which can be effectively applied in sports matters: mediation and arbitration. Mediation and arbitration applied in sport are an effective solution par excellence to problems that arise in the field of sport. Method. For this purpose, a descriptive study of these concepts will be carried out, introducing the different definitions and fundamental principles that govern these procedures. Likewise, a brief analysis of the Mexican legislation on sports matters (General Law of Physical Culture and Sport) will be made, describing the legal regime of mediation and arbitration within Mexican regulations. The nature of the sports appeal and arbitration commission, the Mexican organ of justice, will be described. The commission of appeal and arbitration of the sport, main organ of imparting "sport justice" in Mexico, in its regulations recognizes different types of procedures such as mediation or arbitration, nevertheless to this situation the cases brought before it are very limited. Results. Next, various problems that this commission faces will be presented according to the theories of various jurists specialized in sports matters within Mexico, as well as possible solutions that are proposed to resolve these problems. Conclusions. This commission should have greater powers of coercion in order to function effectively in Mexico, since it does not lack its own binding force and therefore generates a sense of "simulation" in its proceedings.

Key words: Law, Alternative Dispute Resolution, Sports Law.

\section{INTRODUCCIÓN}

La práctica deportiva tiene diversas características y necesidades especiales que hacen necesario contar con métodos específicos y eficientes para solucionar los diversos conflictos que surgen en el ámbito deportivo, por lo que diversos ordenamientos jurídicos han adoptado distintos procedimientos para facilitar y ejecutar con mayor simpleza la resolución de estos conflictos.

Autores como Andreu Camps (2015), señalan que la mediación y el arbitraje son figuras jurídicas que han sido reconocidas desde la Grecia Clásica (VI-IV siglos A.C) y se han convertido en practicas habituales para la resolución de conflictos en nuestros días.

En el artículo 17 de la constitución mexicana se establece que ninguna persona podrá hacer justicia de manera propia, de igual manera instaura el derecho a la administración de justicia por tribunales que deberán seguir los procedimientos de manera pronta, completa e imparcial siempre y cuando no se afecte la igualdad y el debido proceso de las partes. No obstante, el mismo articulo tiene previstos los mecanismos alternativos de solución de controversias ("MASC") mismos que González de Cossío (2003) define como, "herramientas que con frecuencia son utilizadas para solucionar diferencias de manera amistosa y sin la necesidad de tener que recurrir a medios adversariales."

Los principales mecanismos alternos de solución de controversias que se conocen son: la conciliación, la mediación y el arbitraje. La diferencia principal entre estas tres figuras estriba en las facultades o poderes del tercero que interviene en la controversia (Camps, 2016). 
Por lo que el contenido de este trabajo se centrará en los últimos dos, toda vez que son los de mayor aplicación en el terreno deportivo y porque cuentan con legislación reguladora, así como también organismos encargados de su debida aplicación.

\section{CONCEPTOS}

Antes de poder abordar de manera especifica la mediación y el arbitraje deportivo en México, tendremos que establecer los conceptos:

\section{Mediación}

En primer lugar, la mediación se trata de un procedimiento legalmente regulado que tiene como eje principal la voluntariedad y libre decisión de las partes y se desarrolla con la intervención de un profesional neutral que facilita la resolución del conflicto por las propias partes, de forma equitativa, permitiendo el mantenimiento de las relaciones subyacentes y conservando el control sobre el final del conflicto, que quedara expresado en el acuerdo de mediación (Guillén, 2005).

Dentro de la mediación, la persona que funge como mediadora tiene funciones muy especificas tales como: gestionar el procedimiento, encausar a las partes para llegar a una solución, evitar desequilibrio dentro del acuerdo, promover la creación de condiciones positivas para ambas partes, facilitar la comunicación entre las partes, así como generar opciones de solución, pero sin ser el que solucione el conflicto.

La mediación dentro del deporte es un procedimiento idóneo para la solución extrajudicial de los conflictos ocurridos en el deporte. Una de las características que benefician el uso de la mediación para resolver este tipo de conflictos es que un procedimiento judicial convencional carece de especialización deportiva por lo que su resolución suele no ser satisfactoria para las partes.

La flexibilidad de la mediación deportiva como un procedimiento para resolver los conflictos facilita la obtención de acuerdos amistosos, que conducen a soluciones más eficientes y duraderas. De igual manera la mediación deportiva aporta múltiples beneficios a las partes en disputa, pudiendo destacar los siguientes: resolución del conflicto de una manera amistosa, rapidez en la búsqueda de la solución, un costo económico inferior a la vía judicial o al arbitraje, la disponibilidad de una parte mediadora especializada en el sector deportivo, amplia gama de posibles respuestas a la solución del conflicto deportivo.

\section{Arbitraje}

El arbitraje, de acuerdo con Briceño (1995) se define como un proceso jurídico tramitado, desarrollado y resuelto por particulares. Es decir, se habla de una relación jurídica triangular en donde intervienen las dos partes en conflicto un tercero, denominado arbitro que será el encargado de llevar el arbitraje hasta su resolución.

De acuerdo con Maldonado (2017), catedrático de la universidad de Boyacá (Colombia), el arbitraje es uno de los medios de solución de controversias más antiguos que se conocen. Toda vez que autores como Zappalá (2010), señalan que inclusive se confunde con la historia misma del derecho y de la humanidad. Como ejemplo de esto se puede mencionar que ya durante la época del imperio romano, era el pater familias el tercero encargado de dirimir las 
diferencias que se presentaran entre los miembros de la familia; actividad que desarrollaba por medio de un procedimiento saturado de ritos y formalidades. Afirmando Villalba y Moscoso (2008) que dichos procedimientos tenían más relación con el factor religioso que con el jurídico.

Por su parte, el arbitraje deportivo es procedimiento muy utilizado en el sector, pero principalmente en el Fútbol. Este medio tiene como principal característica que las partes implicadas en el conflicto deciden de manera voluntaria someter la controversia a un tercero, mismo que puede ser un solo arbitro o un panel arbitral, el cual decidirá el sentido de la solución a su conflicto. Esté deberá actuar siempre con los principios que rigen el arbitraje, los cuales son: imparcialidad, independencia y confidencialidad.

Otra de las características que distingue al arbitraje, es que en el siempre tendremos la solución al conflicto. Toda vez que al someterse a este medio se obtiene un laudo arbitral, mismo que resulta de obligatorio cumplimiento para las partes, estén o no en conformidad con el resultado.

\section{MEDIACIÓN Y ARBITRAJE EN LA LEGISLACIÓN DEPORTIVA MEXICANA}

\section{A) Mediación}

De acuerdo con la Ley General de Cultura física y deporte (LGCFD) en su articulo 79, la mediación se define como:

"La función de establecer comunicación y negociación entre las partes para prevenir o resolver un conflicto, y por conciliación el método para proponer a las partes alternativas concretas de solución para que resuelvan de común sus diferencias"

La mediación abordará temas de naturaleza estrictamente deportiva, y podrá referirse a actos u omisiones que afecten derechos o prerrogativas de carácter deportivo.

El reglamento de la LGCFD establece un procedimiento para realizar la mediación en sentido deportivo, mismo procedimiento que consta de 4 etapas que serán agotadas para llegar a una solución.

La primera etapa o inicial, consiste en la celebración de un acuerdo de mediación, en el que las partes involucradas acceden a este procedimiento, este acuerdo será elaborado por el Área de Mediación y Conciliación de la Comisión de Apelación y Arbitraje del Deporte, en donde se determine si las etapas se verificarán ante el área de mediación y conciliación de la CAAD o si se designa a un mediador independiente. En esta misma etapa se expondrán las problemáticas y se tendrá contacto con los mediadores.

La segunda etapa es el análisis del caso y construcción de la agenda, en esta etapa se busca identificar los puntos en conflicto, reconocer la responsabilidad de las partes, encontrar similitud entre los intereses y necesidades de los involucrados, de igual manera expone una lista de los temas que son materia del procedimiento. 
Durante la tercera etapa de la mediación se exponen las propuestas de solución, de igual manera se darán a conocer distintas alternativas de solución, se evalúa todo lo anteriormente planteado y se inicia la elaboración de acuerdos para posteriormente iniciar la última etapa.

La etapa final del procedimiento de mediación tiene por objetivo, revisar una ultima vez los acuerdos, antes de elaborar el convenio y someterlo a la aprobación del Pleno de la CAAD y una vez aprobados proceder a la firma del acuerdo.

\section{B) Arbitraje}

González de Cossío (2006) señala que en México "el arbitraje ha sido utilizado en otra rama de controversias que requerían atención eficiente: las controversias en materia deportiva". Afirma que el medio deportivo tiene características y necesidades que hacían necesario contar con un método que les hiciera frente y el arbitraje fue la solución. No obstante, el arbitraje deportivo, es un medio poco utilizado en México. A pesar de que la propia LGCFD y su reglamento cuentan con regulación en la materia, toda vez que entre los años 2010 y 2016 no se realizó ningún procedimiento, de acuerdo con González (2018)

El Reglamento de la LGCFD, establece en su articulo 121 que las controversias jurídicas que se susciten o puedan suscitarse entre deportistas, entrenadores, directivos, autoridades, entidades u organismos deportivos, podrán solucionarse mediante un arbitraje en estricto derecho o en amigable composición.

Por arbitraje en estricto derecho debemos entender que es en el cual las partes fijarán dentro del acuerdo arbitral los puntos esenciales de la controversia y las reglas del procedimiento, acordes con los principios de legalidad, equidad e igualdad entre las partes. Mientras que en el arbitraje en amigable composición las partes fijarán en el acuerdo arbitral las cuestiones que deberán ser objeto de arbitraje y la CAAD tendrá libertad para resolver en conciencia y a buena fe guardada, pero observando las formalidades esenciales del procedimiento.

Continuando con el análisis del arbitraje deportivo, es importante señalar que dentro del Reglamento de la Ley vigente afirma en su artículo 124, que si las particularidades de la controversia jurídica de naturaleza deportiva que se suscite entre deportistas, entrenadores, directivos, autoridades, entidades u organismos deportivos, requieren de una solución inmediata a juicio de las propias partes en conflicto, éstas podrán sujetarse a un arbitraje en amigable composición ante la Comisión de Apelación y Arbitraje del Deporte, que tendrá una duración de 24 horas, dando la opción a duplicar el plazo por la propia CAAD cuando así lo requiera.

De igual manera se afirma que el arbitraje procederá una vez celebrado un acuerdo en donde las partes convengan someter su controversia y aspectos fundamentales ante la CAAD.

En ese acuerdo de deberá, precisar el tipo de arbitraje al que se sujetan y una vez que se resuelva se emitirá el laudo arbitral. Este laudo arbitral emitido por la CAAD deberá cumplimentarse o, en su caso, iniciar su cumplimiento dentro de los 10 días naturales siguientes a la fecha de su notificación, salvo pacto en contrario (artículo 125). 


\section{LA COMISIÓN DE APELACIÓN Y ARBITRAJE DEL DEPORTE}

La Comisión de Apelación y Arbitraje del Deporte (CAAD), fue creada en 1990, con el objeto de contar con una instancia o tribunal de justicia especializada en materia de deporte, dotada de plena jurisdicción y autonomía para dictar sus acuerdos, laudos y resoluciones. Cuyo propósito es resolver controversias entre deportistas, entrenadores, directivos, autoridades, entidades u organismos deportivos. Es decir, conflictos presentados en, durante o con motivo de la práctica del deporte (Meade, 2018).

La CAAD se rige por la LGCFD y esta integrada por un Pleno, mismo que esta conformado por un presidente y cuatro miembros titulares, designados por el poder ejecutivo federal.

$\mathrm{Al}$ igual que otros tribunales, la CAAD se rige por el principio de equidad, inmediatez, imparcialidad, prontitud e igualdad entre las partes. La CAAD además de resolver los conflictos mediante mediación, arbitraje o conciliación, también esta facultada para ser el organismo encargado de resolver el recurso de apelación en materia deportiva. El recurso de apelación es un procedimiento administrativo en forma de juicio, que reviste ciertas formalidades. Las resoluciones emitidas en estos recursos son vinculatorias y obligatorias para las partes.

\section{DISCUSIÓN}

No obstante a las diversas normativas en materia deportiva que se encuentras vigentes en México, es importante reconocer que el derecho deportivo mexicano en referencia a Latinoamérica se ha visto estancado, toda vez que como rama de estudio se ha limitado en esencia, cuenta con la LGCFD y su reglamento, de igual manera cuenta con diversas jurisprudencias emitidas por la Suprema Corte de Justicia, sin embargo aún hace falta crecer para que el sector deportivo en México cuente con verdadera certeza jurídica.

Como se ha mencionado anteriormente, una solución práctica para resolver estos conflictos deportivos son los conocidos medios alternos de solución de controversias, recurrir a los MASC se ha utilizado como una fórmula opcional ante la saturación de casos en los sistemas judiciales, el excesivo formalismo y la falta de confianza en las resoluciones judiciales.

De igual forma Montero (2015) sostiene que "los métodos alternativos se diferencian del procedimiento judicial en distintos aspectos, que los dotan de agilidad y ahorro, en término de costes y tiempo, respecto a la justicia administrada por jueces y tribunales."

Es por esto por lo que los medios alternos de solución de controversias se adecuan a las necesidades del deporte en mayor medida que otros procedimientos (Montesinos, 2012).

No obstante, a que los MASC son una solución efectiva para resolver conflictos deportivos, en México sucede que en la práctica en la CAAD predomina la tramitación del recurso de apelación frente al desarrollo de los MASC antes mencionados (Hernández, 2020).

A lo largo de estos años México ha evolucionado considerablemente en la materia jurídica deportiva. Sin embargo, México ha atravesado diversos problemas en cuestión deportiva, muchos de estos por el desconocimiento relativo a los procedimientos de solución de conflictos deportivos. 
Respecto a la CAAD surge una incógnita ¿Qué debería pasar con ella? Es cierto que el derecho es subjetivo y no es exacto, por lo que responder esto sería interpretativo, no obstante, diversos juristas mexicanos divergen al respecto.

Por ejemplo, González (2018), ex titular de la Comisión de Apelación y Arbitraje del Deporte sugiere la creación de un nuevo régimen jurídico, a través de la creación de una Sala Especializada en materia deportiva adscrita al Tribunal Federal de Justicia Administrativa. Por su parte Camargo (2017), presidente de la Federación Mexicana de Derecho Deportivo y Estudios Legislativos del Deporte, sostiene la opción de crear un Tribunal Federal dependiente del Poder Judicial de la Federación y que tenga la suficiente coercibilidad en sus sentencias, y estas estén sustentadas en derecho. Otro abogado especializado en Derecho Deportivo, Meade (2018), quien ejerció como presidente del Pleno de la Comisión de Apelación y Arbitraje del Deporte en el periodo 2017-2019, considera que es necesario crear reformas a la legislación deportiva así, como otorgar facultades a la CAAD para sancionar a quienes no acaten sus resoluciones.

Respecto a la CAAD se considera fundamental exponer las ideas de diversos expertos en la materia, sin embargo, los medios alternos como el arbitraje son poco utilizados en la CAAD (González, 2018). Esto quiere decir que aún teniendo procedimientos reconocidos en los ordenamientos mexicanos estos son pocos utilizados. Por lo que generar un nuevo tribunal o una sala adscrita sería caer en lo que se busca evitar, una "carga excesiva de trabajo" lo que significaría volver menos efectiva la impartición de justicia deportiva.

Hernández (2020), quien fue miembro de la CAAD en el periodo 2016-2019 sostiene que la CAAD debería transformarse en un Centro de Justicia Alternativa Deportiva que desarrolle los MASC en el ámbito deportivo, permitiendo además la posibilidad de que especialistas externos coadyuven con dicho Centro como árbitros, mediadores y conciliadores privados desarrollando procesos flexibles, dinámicos, de bajo costo y tiempo, así como ad hoc al contexto deportivo.

Finalmente, se considera que la anterior idea resulta más factible, idónea y económica para el estado mexicano, de igual manera se propone elaborar reformas a la LGCFD de tal manera en la que se pueda dotar de mayor eficacia y coercitividad a la Comisión de Apelación y Arbitraje del deporte.

\section{CONCLUSIONES}

Expuesto lo anterior respecto a la mediación y arbitraje en el sector deportivo mexicano, en su aspecto material, sobra decir que hace falta desarrollar el aspecto procesal del derecho deportivo mexicano.

Los medios alternos de solución de controversias tales como la mediación y el arbitraje son una fuente idónea para resolver conflictos de naturaleza deportiva, tanto por la velocidad de resolución como los bajos costos respeto de los procesos judiciales o administrativos.

La CAAD es un organismo funcional que requiere mejoras para una mayor eficacia, sin embargo, es un organismo poco conocido dentro del sector deportivo.

Otra cuestión de la CAAD es la necesidad que tiene de obtener mayores facultades para sancionar a quienes no acaten sus resoluciones. 


\section{REFERENCIAS}

Briseño Sierra, H. (1995). Sobre arbitraje: Estudios. México, Editorial Cárdenas.

Camargo Zamudio, A. (2017, 27 de marzo) La simulación de la Justicia Deportiva en México, Asociación Española de Derecho Deportivo. Recuperado de: https://bit.ly/3qg58Bq

Comité Interinstitucional de Investigación en Cultura Física y Deporte., (2018) "58 Estrategias para la transformación de la Cultura Física Nacional", Escuela Nacional de Entrenadores Deportivos, Recuperado de: https://bit.ly/2I9II3z

Del Carmen Clemente, J; Montenegro González, S; Hernández González, D. (2010) Notas para el estudio del Derecho Deportivo Mexicano (con especial referencia a su aspecto procesal). Revista de la Facultad de Derecho de México. 60, (254), 141- 169.

Díaz Martínez, N. (2017, 25 de Mayo) México y sus avances en la justicia deportiva, iusport. Recuperado de: https://bit.ly/39tkjBn

Flores Fernández, Z. (2019). La cultura física y la práctica del deporte en México. Un derecho social complejo. Revista Cuestiones constitucionales, 1 (40) 185-220.

González Guerrero, N. (2018). Régimen jurídico de la Comisión de Apelación y Arbitraje del Deporte de México y una propuesta de mejora. Universidad de Lleida, España.

González de Cossío, F. (2006). Arbitraje Deportivo. México. Editorial Porrúa.

González de Cossío, F. (2003). Mecanismos alternativos de solución de controversias: nota sobre el desarrollo del área. Ars Iuris. 30: 39 - 67. https://bit.ly/3ok3vAY

Hernández González, D. (2020, 19 de octubre). ¿Cuál podría ser el futuro de la Comisión de Apelación y Arbitraje del Deporte?, Derecho Deportivo mx. Recuperado de: https://bit.ly/2KY4Pes

Ley General de Cultura Física y Deporte, Diario Oficial de la Federación, México, 7 de junio de 2013.

Maldonado Narváez, M., Montaña Cárdenas, L. (2017). Arbitraje: Mecanismo alternativo de solución de conflictos o privatización judicial. JURÍDICAS CUC, 13, (1), 121-146, https://bit.ly/3okGluf

Meade Gutiérrez, E. (2018, 13 de noviembre). La CAAD. Recuperado de: https://bit.ly/3qg5o3t

Montesinos Muñoz, O. (2012). Mediación Deportiva, Revista de Mediación, 5, (10), 6-12.

Comisión Nacional de Cultura Física y Deporte, Mediación. Recuperado 6 de noviembre de 2020, de https://bit.ly/3qqtPez

Pérez Treviño, J. (2019). Resolución de conflictos en el deporte: análisis y propuestas. Madrid, Editorial Reus.

Pérez-Ugena Coromina, M. (2015). Mediación y deporte. Madrid, España: Editorial Dykinson.

Reglamento Ley General de Cultura Física y Deporte México. Diario Oficial de la Federación, México, 23 de mayo de 2014.

Villalba Cuellar J., Moscoso Valderrama, R. (2008). Orígenes y panorama actual del arbitraje. Prolegómenos. Derechos y Valores, XI, ( 22), 141-170

Zappalá, F. (2010). Universalismo histórico del arbitraje. Universitas, 59, (121), 193-216. 